\title{
Role of myocardial hypertrophy on acute and chronic right ventricular performance in relation to chronic volume overload in a porcine model: Relevance for the surgical management of tetralogy of Fallot
}

Thierry Bove, MD, ${ }^{\mathrm{a}}$ Kristof Vandekerckhove, MD, ${ }^{\mathrm{b}}$ Stefaan Bouchez, MD, ${ }^{\mathrm{c}}$ Patrick Wouters, MD, PhD, Pamela Somers, MSc, $\mathrm{PhD},{ }^{\mathrm{d}}$ and Guido Van Nooten, $\mathrm{MD}, \mathrm{PhD}^{\mathrm{a}}$

\begin{abstract}
Objectives: The age for correction of tetralogy of Fallot has progressively declined to the postnatal period, often despite an increased rate of transannular patch repair. However, the long-term effect of premature exposure to chronic pulmonary insufficiency on the right ventricle remains unknown. On the basis of the relationship between the duration of pressure overload and age, the role of previous pressure load-related hypertrophy on right ventricular (RV) performance after chronic volume overload was investigated in a porcine model.
\end{abstract}

\begin{abstract}
Methods: RV hypertrophy (RVH), induced by pulmonary artery banding, was studied in pigs with (RVH plus pulmonary insufficiency [PI]) and without (RVH) subsequent PI. The effect of volume overload was compared between these 2 groups and pigs without RVH but with PI and controls (sham). Both acute and chronic effects on RV function were studied using conductance technology and validated using echocardiography.
\end{abstract}

Results: After chronic volume overload, the end-systolic and end-diastolic volumes were smaller in the $\mathrm{RVH}+\mathrm{PI}$ group than in the PI group, including a lower pulmonary regurgitation fraction $(25 \% \pm 5 \%$ vs $35 \% \pm 5 \% ; P=.002)$. RVH resulted in better preserved systolic function, confirmed by an increased preload recruitable stroke work slope $(14.7 \pm 1.8$ vs $9.3 \pm 1.3 \mathrm{Mw} . \mathrm{s} / \mathrm{mL} ; P=.025)$ and higher RV ejection fraction $(51 \% \pm 3 \%$ vs $45 \% \pm 4 \% ; P=.05)$. Myocardial stiffness was impaired in the RVH+PI group versus the PI group ( $\beta, 0.19 \pm 0.03$ vs $\left.0.12 \pm 0.02 \mathrm{~mL}^{-1} ; P=.001\right)$, presenting restrictive physiology only in the condition associating RVH and PI.

Conclusions: The results of the present study have demonstrated that RVH attenuates the RV remodeling process related to chronic PI. It enables better preservation of contractility but at the cost of sustained diastolic impairment. These findings might help to determine the timing and strategy for repair of tetralogy of Fallot when RV outflow tract morphology indicates a definite need for transannular reconstruction. (J Thorac Cardiovasc Surg 2014;147:1956-65)

Despite more than 50 years of experience with the surgical management of tetralogy of Fallot (TOF), the optimal timing and surgical approach are still a matter of debate. During the past decade, primary repair has been advanced to the first months of life and even the neonatal age, without compromising the surgical outcome in terms of mortality. ${ }^{1-3}$

From the Department of Cardiac Surgery, ${ }^{a}$ University Hospital of Ghent, Ghent, Belgium; Department of Pediatric Cardiology, ${ }^{b}$ University Hospital of Ghent, Ghent, Belgium; Department of Anesthesiology, ${ }^{\mathrm{c}}$ University Hospital of Ghent, Ghent, Belgium; and Department of Histology and Human Pathology, ${ }^{\mathrm{d}}$ University Hospital of Ghent, Ghent, Belgium.

This study was supported by a grant from the Fonds voor Hartchirurgie-Fondation pour la Chirurgie Cardiaque, Belgium.

Disclosures: Authors have nothing to disclose with regard to commercial support.

Received for publication July 31, 2013; revisions received Oct 3, 2013; accepted for publication Oct 11, 2013; available ahead of print Nov 25, 2013.

Address for reprints: Thierry Bove, MD, Department of Cardiac Surgery, University Hospital of Ghent, De Pintelaan 185, 5K12, Ghent 9000, Belgium (E-mail: thierry.bove@ugent.be).

0022-5223/\$36.00

Copyright $($ c 2014 by The American Association for Thoracic Surgery

http://dx.doi.org/10.1016/j.jtcvs.2013.10.026
In addition to avoiding the use of a palliative shunt and its potential complications, protagonists have claimed the physiologic benefit of the early elimination of hypoxemia with its adverse effects on organ maturation, improved pulmonary angiogenesis and alveologenesis, and reduced right ventricular (RV) hypertrophy (RVH). A major concern has been the high frequency of transannular patch (TAP) reconstruction of the RV outflow tract (RVOT), subjecting these young children prematurely to the physiologic consequences of pulmonary insufficiency (PI). Hence, the long-term effects on RV function need to be known.

Pressure overload usually results in compensatory myocardial hypertrophy. Previous work has shown improved contractile performance of the right ventricle by gradual and chronic afterload increase but at the cost of impaired diastolic function. ${ }^{4,5}$ This ambivalent phenomenon must be considered because of its effect on RV performance when challenged by acute and chronic PI-related volume overload as a common sequela of TOF 

Abbreviations and Acronyms
BSA = body surface area
$\mathrm{PAB}=$ pulmonary artery banding
PI = pulmonary insufficiency
$\mathrm{PRF}=$ pulmonary regurgitation fraction
$\mathrm{RVEF}=$ right ventricular ejection fraction
$\mathrm{RVH}=$ right ventricular hypertrophy
RVOT $=$ right ventricular outflow tract
$\mathrm{TAP}=$ transannular patch
TOF $=$ tetralogy of Fallot
TTE $=$ transthoracic echocardiography

repair. Because a direct relationship exists between patient age and the extent of $\mathrm{RVH}$, insight into this process might help the decision-making process concerning the timing and surgical management of TOF with regard to the RVOT morphology.

The purpose of the present study was to mimic the physiologic conditions of TOF repair with postoperative PI in a growing swine model. We first induced RV pressure overload to develop RVH, followed by chronic volume overload using surgical TAP reconstruction of the RVOT. The effect of RVH on RV remodeling and function was hemodynamically assessed using the conductance technique for quantification of the RV volumes and preload-independent indexes of systolic and diastolic function.

\section{METHODS}

The study protocol was performed according to the standards of the "Guide for the Care and Use of Laboratory Animals" (National Institutes of Health, publication no. 85-23, revised 1996) and approved by the ethical committee for animal research of the Ghent University Hospital (ECD 11/27).

\section{Study Protocol}

A total of 28 Landrace pigs were entered into the study protocol. These were divided into 4 groups: (1) RVH+PI group $(\mathrm{n}=7)$ with pressure overload-induced RVH and subsequent volume overload by TAP, (2) RVH group $(n=7)$ with isolated pressure overload by pulmonary artery banding $(\mathrm{PAB})$ and secondary relief of pulmonary artery obstruction, (3) PI group $(\mathrm{n}=7)$ with volume overload by TAP-mediated PI only, and (4) a sham group $(n=7)$ as the control group, without exposure to any pathophysiologic loading condition.

Of the 28 pigs, 14 underwent gradual pressure overload with a loose $\mathrm{PAB}$ at 6 to 7 weeks of age (mean weight, $17.9 \pm 1.8 \mathrm{~kg}$ ). Follow-up examinations using transthoracic echocardiography (TTE) was done for serial evaluation of RV function and PAB gradient. After $\sim 8$ weeks, the pigs were equally and randomly, divided into the RVH+PI and $\mathrm{RVH}$ groups. One pig from each group died during the study period and was excluded. All the pigs underwent evaluation of RV function using the conductance catheter at the acute, postoperative phase and late, chronic phase, representing an interval of $\sim 3$ months (mean, $13.1 \pm 1.4$ weeks). According to previous work, ${ }^{6,7}$ this interval was determined by the rapid organ maturation and growth of the Landrace swine, corresponding roughly to mid-term expectancy in humans. The pigs in the PI and sham groups were age-matched to the former groups at the first hemodynamic assessment. TTE was performed within the same week of the hemodynamic investigation for assessment of the procedural effect on the right ventricle. The conduct of the study protocol is shown in Figure 1.

\section{Operative Procedures}

The anesthesia protocol and basic hemodynamic monitoring of all surgical procedures was identical to those used in a previous study. ${ }^{6}$

$\mathrm{PAB}$ was performed through a small left thoracotomy and encircling the pulmonary artery with a 5-mm-large band of silicone elastomere (Perthese, Bornel, France) to obtain a gradient of $\geq 10 \mathrm{~mm} \mathrm{Hg}$, with the systemic blood pressure remaining stable. Additional growth of the pig should result in progressive afterload increase. The hemodynamic effect of $\mathrm{PAB}$ was assessed by direct measurement of the RV pressure in relation to the aortic pressure and distal pulmonary artery pressure.

The second surgical step, done at phase 1, included redo-thoracotomy for removal of the PAB and resection of the surrounding fibrous tissue, which mostly relieved the obstruction effectively. In 3 pigs, an additional patch plasty of the pulmonary trunk was needed to obtain a residual target gradient of $<10 \mathrm{~mm} \mathrm{Hg}$. To create significant PI, a longitudinal incision across the ventriculoarterial junction was made under partial clamping of the RVOT, the anterior pulmonic valve leaflet was excised, and a transannular polytetrafluoroethylene patch (Gore-Tex, W. L. Gore \& Assoc, Inc, Newark, Del), 30 by $20 \mathrm{~mm}$, was inserted. In the RVH group, no other surgery than PAB removal was done. In the PI group, PI was obtained with the first procedure just as in the RVH+PI group. The sham-operated pigs underwent left thoracotomy for hemodynamic testing, without RV surgery.

At the end of each procedure, the pigs were extubated and treated with intramuscular buprenorphine $0.03 \mathrm{~mL} / \mathrm{kg}$ and intercostal bloc with levobupivacaine $5 \mathrm{mg} / \mathrm{kg}$.

At the last phase, cardiac exposure was obtained solely for hemodynamic testing through a median sternotomy, with release of the pericardial adhesions.

\section{Hemodynamic Study With Conductance Catheter}

In the acute phase, a 7F dual-field, pressure-volume catheter (CD Leycom, Zoetermeer, The Netherlands) was introduced into the right ventricle from the RVOT directed toward the apex. Correct catheter positioning was confirmed by fluoroscopy. The catheter was connected to a Sigma-M module and digitized at $250 \mathrm{~Hz}$ for on-line computer analysis with the Conduct NT-CFL-512 software (CD Leycom). Preload modulation at phase 1 was achieved using radioscopically guided placement of a pulmonary artery balloon catheter (PTS-303 NUMED, Heart Medical, Best, The Netherlands) into the inferior vena cava by way of a puncture in the right jugular vein. During phase 2, the performance of the conductance measurements was similar but inferior vena cava occlusion was done surgically using a tourniquet.

The pressure and volume data were obtained at end-expiration, using the method from previous experiments. ${ }^{6}$ Volume calibration was performed by integration of slope factor $\alpha$ for cardiac output and verified for effective cardiac output by pulse-pressure waveform analysis of the aortic pressure signal (Vigileo, Edwards Lifesciences, Irvine, Calif). Calibration for parallel conductance was acquired during injection of $0.02 \mathrm{~mL} / \mathrm{kg}$ hypertonic saline. The baseline measurements included the end-systolic and end-diastolic RV volumes, with subsequent calculation of the stroke volume and ejection fraction. To correct for growth variability between the pigs among the groups, volume-dependent variables were indexed to the body surface area (BSA), using the equation for swine ${ }^{7}$ : BSA $\left(\mathrm{cm}^{2}\right)=734 \times$ weight $(\mathrm{kg})^{0.656}$.

As described previously, ${ }^{8}$ the pulmonary regurgitation fraction (PRF) was quantified as the ratio of the volume increase during pressure decline to stroke volume during 1 cardiac cycle at baseline (Figure 2). According to the instantaneous pressure-volume relationship changes during transient 


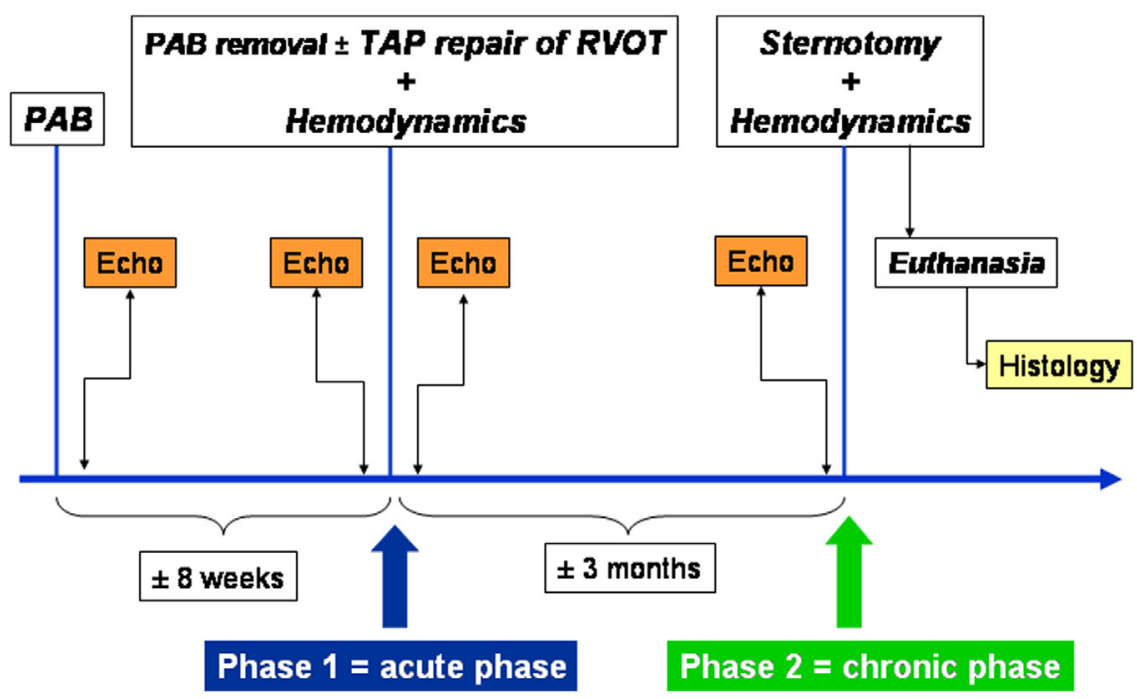

FIGURE 1. Time course and conduct of the study, defining the surgical procedure and right ventricular function assessment at phases 1 and 2. $P A B$, Pulmonary artery banding; TAP, transannular patch; RVOT, right ventricular outflow tract; Echo, echocardiography.

occlusion of the inferior vena cava, RV contractility was quantified by the slope $-\left(\mathrm{M}_{\mathrm{w}}\right)$ of the preload-recruitable stroke work relation and the slope- $\left(\mathrm{E}_{\max }\right)$ of the end-systolic pressure-volume relationship. The volume intercept of the end-systolic pressure-volume relationship was determined at a pressure level of $25 \mathrm{~mm} \mathrm{Hg}$ and indexed to the BSA $\left(\mathrm{V}_{25}-\mathrm{I}\right)$. Only recordings with a $<10 \%$ change in the heart rate and a correlation coefficient of the linear regression $>0.90$ were eligible. Evaluation of the diastolic RV function was determined solely from the passive ventricular compliance, because active isovolumetric relaxation was not reliable in the groups with PI. RV compliance is expressed as the chamber stiffness constant $\beta$, derived from the end-diastolic pressure-volume relationship.

\section{Echocardiography}

TTE was performed using the portable Vivid-I ultrasound system (General Electric Healthcare, Buckinghamshire, UK) and a $3-\mathrm{MHz}$ transducer by a single observer (K.V.D.K.), who was unaware of the invasive hemodynamic results. The primary goal of TTE was to validate the functional effect of each surgical procedure. The timing of each examination is indicated in Figure 1. Echocardiography included 2-dimensional anatomic imaging of the right ventricle for size estimation at the basal, midventricular, and RVOT level. RVH was quantified by measurement of the RV free wall and interventricular septal thickness, adjusted for the BSA. The tricuspid and pulmonary valves were evaluated using color flow mapping, and the degree of regurgitation was semiquantitatively nominated as grade 1 to 4 , according to standard criteria. Tricuspid inflow signal analysis included determination of the E-wave and A-wave velocity, E-/A-wave velocity ratio, E-wave deceleration time, and E-wave deceleration slope. Pulse-wave Doppler analysis of the main pulmonary flow was performed to (1) estimate the flow velocity over the RVOT to follow the gradient during PAB and to exclude residual obstruction after debanding; and (2) calculate the duration of the diastolic regurgitant jet in the presence of PI. The presence of an A-wave at late diastole throughout the respiratory cycle was categorized as a pattern of restrictive physiology. Estimation of systolic RV function was determined from the tricuspid

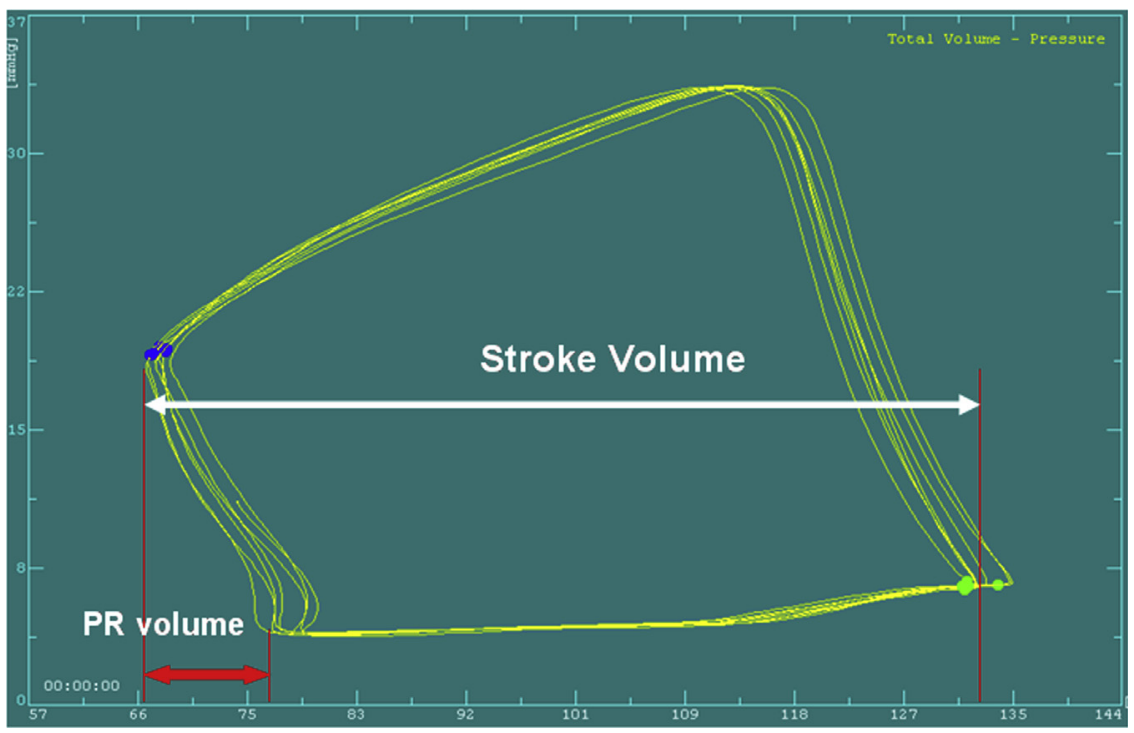

FIGURE 2. Baseline pressure-volume loop of right ventricle in pig in the right ventricular hypertrophy and pulmonary insufficiency group, showing the method of pulmonary regurgitation $(P R)$ fraction calculation $(\mathrm{PR}$ volume/stroke volume $\times 100)$. 
annulus plane systolic excursion for longitudinal RV function and fractional shortening measurement at the midventricular level on M-mode for radial RV function.

\section{Euthanasia and Histopathologic Examination}

At the end of the study, the pigs were killed with an intravenous solution of embutramide $200 \mathrm{mg}$, mebenzonium iodide $200 \mathrm{mg}$, tetracaine hydrochloride $5 \mathrm{mg}$, dimethylformamide $1 \mathrm{mg}$ (T61) at a dose of $0.3 \mathrm{~mL} / \mathrm{kg}$. The heart was harvested and detached from the atria and great vessels; the ventricles were divided into the free wall of the right and left ventricles and the interventricular septum. Each component was weighted separately to calculate the $\mathrm{RV} /$ heart ratio and $\mathrm{RV}$ mass/end-diastolic volume ratio, adjusted for the BSA. RV and LV tissue samples were taken for histologic analysis, with hematoxylin-eosin and Masson's trichrome staining for delineation of the interstitial collagen extent. The myocyte cell area was counted to determine the presence of hypertrophy, including the presence of cell apoptosis.

\section{Statistical Analysis}

All data are expressed as the mean \pm standard deviation. Data distribution was tested for normality using Shapiro-Wilks testing. Normally distributed data were compared among groups using 1-way analysis of variance, with Tukey or Dunnett-T3 correction for multiple comparisons, depending on the homogeneity of variance. For nonparametric data, group comparisons were made using Kruskal-Wallis analysis and subsequent Mann-Whitney testing with Bonferroni's correction. A paired $t$ test or Wilcoxon signed rank test was used to compare the effect of the PAB in terms of gradient and RV wall thickness by echocardiography between the 2 measurement points in the pigs with PAB-induced pressure overload.

The correlation between the conductance-related and echocardiographic observations was based on the Pearson or Spearman rank test, as appropriate. Statistical analysis was done using the Statistical Package for Social Sciences, version 19.0, software (SPSS, Inc, Chicago, Ill). $P<.05$ was considered significant.

\section{RESULTS}

\section{Validation of RVH by PAB-Induced Pressure Overload}

PAB caused a comparable postoperative gradient and $\mathrm{RV} / \mathrm{LV}$ pressure ratio in the $\mathrm{RVH}+\mathrm{PI}$ and $\mathrm{RVH}$ groups $(12.5 \pm 2.0$ vs $14.1 \pm 6.1 \mathrm{~mm} \mathrm{Hg}, P=.57$; and $51 \% \pm 6 \%$ vs $52 \% \pm 5 \%, P=.64$; respectively). This corresponded to an echocardiographic gradient of $26.5 \pm 10.1$ and $26.3 \pm 15.4 \mathrm{~mm} \mathrm{Hg}(P=.97)$. After 8 weeks, the corresponding echocardiographic gradients had increased to $63.9 \pm 14.4$ and $52.8 \pm 18.8 \mathrm{~mm} \mathrm{Hg}(P=.28)$. RVH was confirmed by a significant and comparable increase of the RV wall thickness in both groups (free wall thickness, $5.9 \pm 1.2$ to $9.3 \pm 1.7 \mathrm{~mm}$, $P=.005$; and interventricular septal thickness, $6.8 \pm 1.4$ to $10.2 \pm 2.5 \mathrm{~mm}, P=.007)$. Both PAB groups had increased free wall thickness compared with the non-PAB groups, as shown by TTE at phase $1(\mathrm{RVH}+\mathrm{PI}$ group, $11.0 \pm 1.4 \mathrm{~mm}$; $\mathrm{RVH}$ group, $10.8 \pm 1.8 \mathrm{~mm}$; sham group, $7.6 \pm 0.6 \mathrm{~mm}$; and PI group, $7.0 \pm 0.4 \mathrm{~mm} ; P=.002$ ).

\section{RV Volume- and Pressure-Related Results}

The PRF was greater in the PI group than in the RVH+PI group at the acute stage (Table 1). Because the PRF had increased proportionally after 3 months in both groups, the late difference in PRF remained significant. This had an immediate effect on the RV volume, resulting in a smaller indexed RV end-systolic and end-diastolic volume in the RVH+PI group compared with the PI group in the acute phase. This was equally reflected in the chronic phase, with a less dilated right ventricle in the $\mathrm{RVH}+\mathrm{PI}$ group than in the PI group. Both volume-overloaded groups had a larger right ventricle than the RVH and sham groups.

$\mathrm{RV}$ pump function was globally preserved in all groups at phase 1 , reaching only a small significant difference in the $\mathrm{RV}$ ejection fraction (RVEF) between the PI and RVH groups $(62 \% \pm 3 \%$ vs $69 \% \pm 5 \%, P=.009)$. After 3 months, the RVEF had worsened significantly in the PI group than in other groups; however, the RVH+PI group also had a lower RVEF than did the sham group (mean difference, $8 \% \pm 2 \%, P=.008$ ). The global RV function was comparable between the RVH and sham groups regarding RVEF at both phases but included a smaller indexed RV end-systolic and end-diastolic volume for the RVH group in the acute phase.

The groups with previous pressure overload had greater $\mathrm{RV}$ end-systolic and end-diastolic pressure at phase 1 . At the chronic stage, only the RV end-diastolic pressure remained significantly elevated in these groups, although the RV end-systolic pressure had equalized. Pulmonic valve dysfunction resulted in a lower pulmonary artery end-diastolic pressure in the PI group. However, the pulmonary artery end-diastolic pressure remained comparable between the RVH+PI group and the RVH and sham groups in the acute phase, despite significant PI. This phenomenon was identical after 3 months, indicating a significantly lower pulmonary artery end-diastolic pressure in the RVH+PI group. Finally, the stroke work was significantly increased in all study groups compared with the sham group at phase 1 . Three months later, no difference was found in the stroke work between the sham and $\mathrm{RVH}$ groups, probably owing to regression of the RV end-systolic pressure. Chronic volume overload led to increased stroke work in the RVH+PI group and even more in the PI group compared with the sham group.

\section{Assessment of Load-Independent Systolic and Diastolic RV Performance}

Contractile function was mainly impaired in the PI group, illustrated by a lower $E_{\max }$ and $\mathrm{M}_{\mathrm{w}}$ slope at phase 1 , although the difference with the RVH+PI group was not significant. At phase 2, both contractility indexes evolved in disadvantages to both groups with volume overload. The preload recruitable stroke work relation slope was significantly lower in the PI group than in the other groups and was also significantly decreased compared with that in the RVH+PI group. Analysis of the $\mathrm{E}_{\max }$ and volume-intercept $\mathrm{V}_{25}$-I showed a similar trend; however, 
TABLE 1. RV volume and pressure data

\begin{tabular}{|c|c|c|c|c|c|}
\hline Variable & $\mathbf{R V H}+\mathbf{P I}$ & RVH & PI & Sham & $P$ value \\
\hline \multicolumn{6}{|l|}{ Weight (kg) } \\
\hline Acute & $39 \pm 8$ & $44 \pm 4$ & $32 \pm 4$ & $31 \pm 12^{*}$ & .02 \\
\hline Chronic & $102 \pm 16$ & $109 \pm 4$ & $89 \pm 11$ & $77 \pm 19^{*}$ & .002 \\
\hline \multicolumn{6}{|l|}{$\operatorname{BSA}\left(m^{2}\right)$} \\
\hline Acute & $0.81 \pm 0.11$ & $0.88 \pm 0.05$ & $0.71 \pm 0.06$ & $0.69 \pm 0.17 *$ & .02 \\
\hline Chronic & $1.52 \pm 0.16$ & $1.60 \pm 0.03$ & $1.39 \pm 0.12$ & $1.26 \pm 0.21^{*}$ & .002 \\
\hline \multicolumn{6}{|c|}{ Heart rate (beats/min) } \\
\hline Acute & $96 \pm 7$ & $93 \pm 6$ & $96 \pm 4$ & $90 \pm 4$ & .11 \\
\hline Chronic & $78 \pm 3$ & $81 \pm 3$ & $78 \pm 9$ & $79 \pm 5$ & .77 \\
\hline \multicolumn{6}{|l|}{ PRF (\%) } \\
\hline Acute & $19 \pm 4$ & - & $26 \pm 4$ & - & .02 \\
\hline Chronic & $25 \pm 5$ & - & $35 \pm 5$ & - & .002 \\
\hline$\% \Delta$ & $29 \pm 4$ & - & $39 \pm 21$ & - & .3 \\
\hline \multicolumn{6}{|l|}{ RVESP (mm Hg) } \\
\hline Acute & $33 \pm 4$ & $32 \pm 3$ & $26 \pm 4$ & $24 \pm 5$ & $.001 \dagger$ \\
\hline Chronic & $25 \pm 3$ & $27 \pm 5$ & $19 \pm 5$ & $21 \pm 6$ & .05 \\
\hline \multicolumn{6}{|l|}{ RVEDP (mm Hg) } \\
\hline Acute & $14 \pm 1$ & $15 \pm 3$ & $10 \pm 2$ & $8 \pm 3$ & $<.001 \dagger$ \\
\hline Chronic & $11 \pm 1$ & $12 \pm 1$ & $6 \pm 2$ & $7 \pm 3$ & $<.001 \dagger$ \\
\hline \multicolumn{6}{|l|}{ PAESP $(\mathrm{mm} \mathrm{Hg})$} \\
\hline Acute & $29 \pm 4$ & $28 \pm 4$ & $27 \pm 4$ & $24 \pm 5$ & .16 \\
\hline Chronic & $25 \pm 3$ & $25 \pm 5$ & $19 \pm 5$ & $22 \pm 7$ & .17 \\
\hline \multicolumn{6}{|l|}{ PAEDP $(\mathrm{mm} \mathrm{Hg})$} \\
\hline Acute & $18 \pm 3$ & $18 \pm 2$ & $10 \pm 2 \ddagger$ & $17 \pm 5$ & $<.001$ \\
\hline Chronic & $11 \pm 2$ & $15 \pm 2$ & $8 \pm 1 \ddagger$ & $14 \pm 3$ & $<.001$ \\
\hline \multicolumn{6}{|l|}{$\operatorname{ESVI}\left(\mathrm{mL} / \mathrm{m}^{2}\right)$} \\
\hline Acute & $40 \pm 3$ & $27 \pm 5$ & $46 \pm 4$ & $34 \pm 4$ & $<.001 \S$ \\
\hline Chronic & $62 \pm 5$ & $51 \pm 4$ & $76 \pm 7$ & $43 \pm 6$ & $<.001 \|$ \\
\hline \multicolumn{6}{|l|}{$\operatorname{EDVI}\left(\mathrm{mL} / \mathrm{m}^{2}\right)$} \\
\hline Acute & $111 \pm 4$ & $88 \pm 8$ & $123 \pm 9$ & $101 \pm 5$ & $<.001 \S$ \\
\hline Chronic & $127 \pm 3$ & $111 \pm 5$ & $140 \pm 7$ & $103 \pm 9$ & $<.001 \|$ \\
\hline \multicolumn{6}{|l|}{$\mathrm{SVI}\left(\mathrm{mL} / \mathrm{m}^{2}\right)$} \\
\hline Acute & $71 \pm 4$ & $61 \pm 7$ & $76 \pm 8$ ฯ & $66 \pm 5$ & .001 \\
\hline Chronic & $65 \pm 4$ & $60 \pm 3$ & $64 \pm 7$ & $61 \pm 6$ & .38 \\
\hline \multicolumn{6}{|c|}{$\mathrm{SWI}\left(\mathrm{mL} / \mathrm{mm} \mathrm{Hg} / \mathrm{m}^{2}\right)$} \\
\hline Acute & $1244 \pm 207$ & $1032 \pm 370$ & $1004 \pm 192$ & $777 \pm 148 \#$ & .02 \\
\hline Chronic & $1090 \pm 87$ & $752 \pm 110$ & $1222 \pm 214$ & $813 \pm 147$ & $<.001^{* *}$ \\
\hline \multicolumn{6}{|l|}{ RVEF (\%) } \\
\hline Acute & $64 \pm 3$ & $69 \pm 5$ & $62 \pm 3+\dagger$ & $66 \pm 3$ & .01 \\
\hline Chronic & $51 \pm 3$ & $54 \pm 3$ & $45 \pm 4$ & $59 \pm 4$ & $<.001^{* *}$ \\
\hline \multicolumn{6}{|c|}{ Effective CI $\left(\mathrm{L} / \mathrm{min} / \mathrm{m}^{2}\right)$} \\
\hline Acute & $6.1 \pm 0.4$ & $5.0 \pm 0.7$ & $6.2 \pm 0.6$ & $5.1 \pm 0.6$ & $<.001^{* *}$ \\
\hline Chronic & $3.8 \pm 0.2$ & $4.0 \pm 0.3$ & $3.7 \pm 0.4$ & $3.5 \pm 0.8$ & .45 \\
\hline
\end{tabular}

Analysis of variance used for all $P$ values; post hoc Tukey correction for multiple comparisons. $R V H$, Right ventricular hypertrophy; $P I$, pulmonary insufficiency; $B S A$, body surface area; $P R F$, pulmonary regurgitation fraction; $R V E S P$, RV end-systolic pressure; $R V E D P$, RV end-diastolic pressure; $P A E S P$, pulmonary artery end-systolic pressure; $P A E D P$, pulmonary artery end-diastolic pressure; ESVI, indexed RV end-systolic volume; EDVI, indexed RV end-diastolic volume; $S V I$, indexed stroke volume; SWI, indexed stroke work; $R V E F$, RV ejection fraction; $C I$, cardiac index. ${ }^{*} P<.05$ between the $\mathrm{RVH}$ and sham groups. $\dagger P<.05$ for all comparisons except between the RVH and RVH+PI and the PI and sham groups. $\ddagger P<.05$ between the PI group and other groups. $\S P<.05$ for all comparisons except between the sham and RVH+PI groups. $\| P<.05$ for all comparisons except between the sham and RVH groups. $₫ P<.05$ between the PI and sham and RVH groups. $\# P<.05$ between the sham and RVH+PI groups. **P $P .05$ for all comparisons except between the sham and RVH groups and the PI and RVH+PI groups. $\dagger \dagger P<.05$ between the PI and RVH groups.

the difference between the PI and RVH+PI groups at phase 2 did not reach statistical significance $(P=.09)$. Contractility was comparable between the RVH and sham groups in the acute and chronic phases.

RV compliance was significantly altered by hypertrophy, with a significantly increased stiffness coefficient $\beta$ in the $\mathrm{RVH}+\mathrm{PI}$ and $\mathrm{RVH}$ groups at phase 1 that persisted after 3 months. However, the diastolic compliance coefficient $\beta$ appeared to be lower in all groups at phase 2 . This could be explained by the different measurement conditions, minimizing the effect of pericardial restraint by using sternotomy for cardiac exposure. The preload 


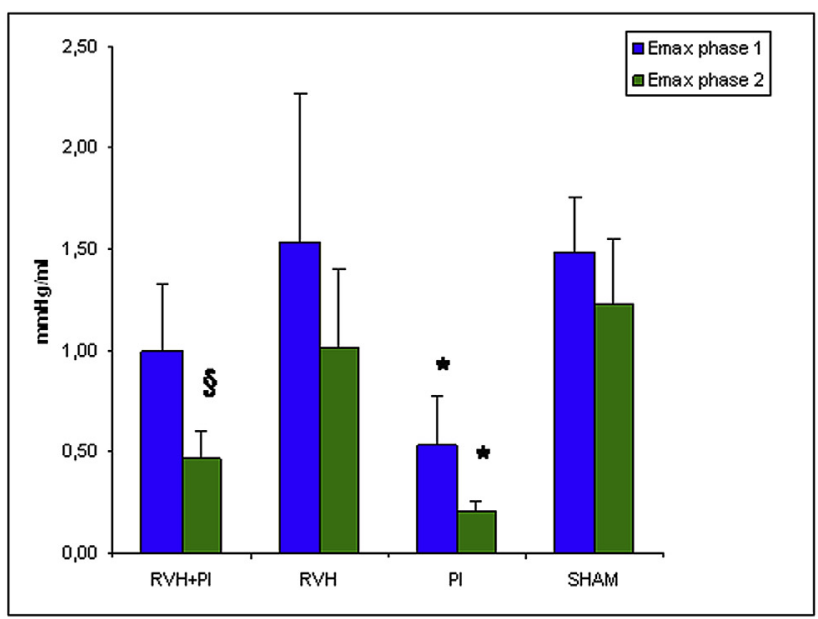

\begin{tabular}{|c|c|c|c|c|c|}
\hline & $\underline{\mathbf{R H}+\mathbf{P I}}$ & RMH & Pl & SHAM & p.value \\
\hline \multicolumn{6}{|c|}{ Emax finnHginn } \\
\hline acute & $1.00 \pm 0.33$ & $1.54 \pm 0.72$ & $0.53 \pm 0.24^{\star}$ & $1.49 \pm 0.27$ & 0.001 \\
\hline chronic & $0.47 \pm 0.13^{\hat{s}}$ & $1.02 \pm 0.39$ & $0.21 \pm 0.04^{*}$ & $1.23 \pm 0.33$ & $<0.001$ \\
\hline \multicolumn{6}{|l|}{ V25+ (nntimn]) } \\
\hline acute & $42 \pm 11$ & $31 \pm 10$ & $46 \pm 9^{\star}$ & $34 \pm 9$ & 0.03 \\
\hline chronic & $52 \pm 15^{\S}$ & $45 \pm 12$ & $61 \pm 13^{*}$ & $31 \pm 5$ & 0.001 \\
\hline
\end{tabular}

A

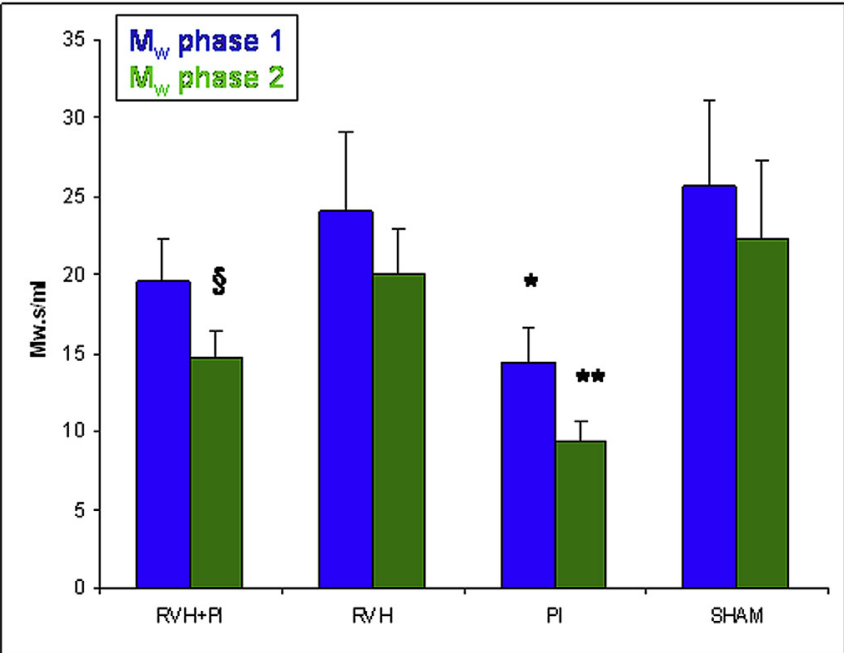

\begin{tabular}{|c|c|c|c|c|c|}
\hline & $\underline{\mathrm{R} M \mathrm{H}+\mathrm{PI}}$ & RMH & $\underline{\text { PI }}$ & SHAM & p-value \\
\hline \multicolumn{6}{|c|}{ PRSW slape Ahw.skinl] } \\
\hline acute & $19.5 \pm 2.8$ & $24.1 \pm 5.1$ & $14.4 \pm 2.2^{*}$ & $25.7 \pm 5.6$ & $<0.001$ \\
\hline chronic & $14.7 \pm 1.8^{8}$ & $20.0 \pm 2.9$ & $9.3 \pm 1.3^{m}$ & $22.4 \pm 4.9$ & $<0.001$ \\
\hline
\end{tabular}

B

FIGURE 3. A, Bar plot of $\mathrm{E}_{\max }$ derived from end-systolic pressure-volume relationship at both study phases. ${ }^{*} P<.05$ between the pulmonary insufficiency $(P I)$ group and the sham and right ventricular hypertrophy $(R V H)$ groups; $\S P<.05$ between the $\mathrm{RVH}+\mathrm{PI}$ group and sham and $\mathrm{RVH}$ groups. $\mathrm{B}, \mathrm{Bar}$ plot of $\mathrm{M}_{\mathrm{w}}$ derived from preload recruitable stroke work at both study phases. $* P<.05$ between the PI group and sham and RVH groups; ** $P<.05$ between the PI group and all other groups; $\S P<.05$ between the RVH+PI group and sham and RVH groups.

independent RV function indexes are depicted in Figures 3 and 4.

\section{Echocardiographic Data}

Echocardiography at phase 1 confirmed the presence of significant PI in the RVH+PI and PI groups, with a shorter PI duration $(256 \pm 14$ and $291 \pm 10 \mathrm{~ms}$, respectively; $P=.01$ ) in the $\mathrm{RVH}+\mathrm{PI}$ group (Table 2 ). After 3 months, both groups had a larger RV size at the subpulmonary level; however, the differences at the basal and midventricular level were less pronounced. Again, the PI duration was significantly shorter in the 

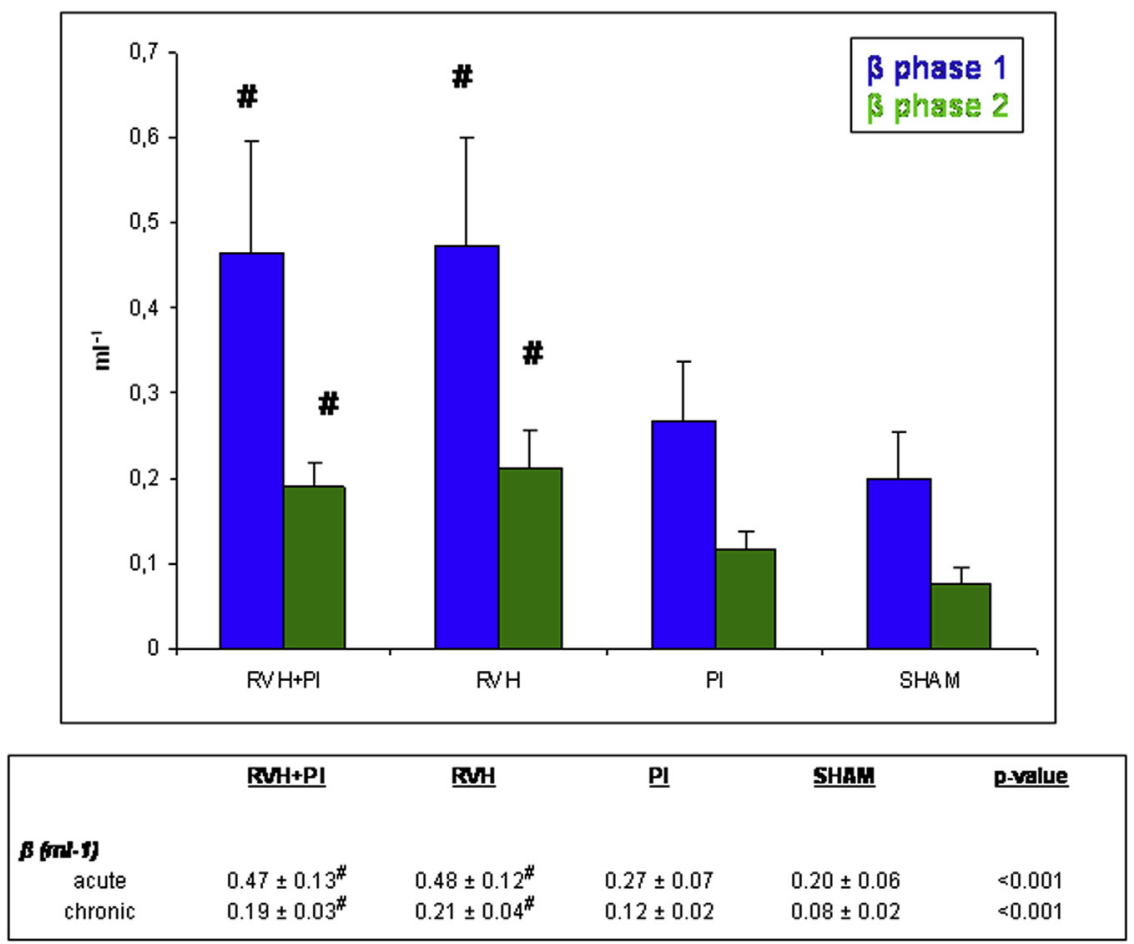

FIGURE 4. Bar plot of $\beta$-stiffness coefficient derived from end-diastolic pressure-volume relationship at both study phases. \#P<.05 between the right ventricular hypertrophy $(R V H)$ and RVH plus pulmonary insufficiency $(P I)$ groups and sham and PI groups.

RVH+PI group than in the PI group $(263 \pm 19$ vs $293 \pm 9 \mathrm{~ms} ; P=.047)$.

Restrictive physiology was seen mainly in the RVH+PI group in 4 of the 6 pigs in phase 1 and 5 pigs in at phase 2. This was only noted at phase 1 in 1 pig in the RVH group and had disappeared by phase 2 . Regarding diastolic function, RVH was only associated with a significant decrease of the E-wave deceleration slope compared with the sham group. This parameter correlated inversely with the stiffness coefficient $\beta(\rho=-0.70 ; P=.001)$ and RV end-diastolic pressure $(\rho-0.53 ; P=.026)$ at the chronic phase. In contrast, the echocardiographic determinants of systolic RV function, such as fractional shortening ( $\rho=0.05 ; P=.84)$ and tricuspid annulus plane systolic excursion $(\rho=0.16 ; P=.52)$, were poorly associated with the systolic indexes obtained using the conductance technique. Echocardiographic RV size estimation showed a good correlation between the indexed RV end-diastolic volume and RV size at the basal level $(\rho=0.49$; $P=.04)$, midventricular level $(\rho=0.34 ; P=.07)$, and RVOT level $(\rho=0.55 ; P=.02)$.

\section{Histopathologic Results}

A significant difference in the $\mathrm{RV}$ mass was found among all groups $\left(\mathrm{RVH}+\mathrm{PI}, 99 \pm 10 \mathrm{~g} / \mathrm{m}^{2}\right.$; PI, $87 \pm 5 \mathrm{~g} / \mathrm{m}^{2}$; RVH, $72 \pm 3 \mathrm{~g} / \mathrm{m}^{2}$; sham, $\left.50 \pm 1 \mathrm{~g} / \mathrm{m}^{2} ; P<.03\right)$. The $\mathrm{RV}$ mass/total heart weight ratio was greater in the volume-overloaded groups $(\mathrm{RVH}+\mathrm{PI}, \quad 36 \% \pm 5 \%$;
PI, $38 \% \pm 2 \%$; RVH, $29 \% \pm 1 \%$; sham, $26 \% \pm 2 \%$; $P=.001)$. The RV mass/volume ratio was particularly increased in the RVH+PI group $(0.77 \pm 0.08 \mathrm{~g} / \mathrm{mL})$ compared with the PI group $(0.62 \pm 0.07 \mathrm{~g} / \mathrm{mL}$, $P=.003)$ and RVH group $(0.64 \pm 0.04 \mathrm{~g} / \mathrm{mL}, P=.012)$. In contrast, the sham group had the lowest ratio $(0.51 \pm 0.05 \mathrm{~g} / \mathrm{mL}, P<.001)$, which was also lower than that in the PI group $(P=.05)$ and RVH group $(P=.03)$. Microscopic examination of the right ventricle revealed evident cellular hypertrophy and focal apoptosis in the $\mathrm{RVH}+\mathrm{PI}$ and RVH groups, more than in the PI group. Interstitial fibrosis and myocardial disarray were equally observed in all treatment groups but to a greater extent in the RVH+PI group. The microscopic examination findings were normal in the sham group.

\section{DISCUSSION}

In the present study, a physiologic model was established to investigate the role of $\mathrm{RVH}$, initiated by previous pressure overload, on RV performance during acute and chronic exposure to PI-related volume overload in growing swine. To our knowledge, such an experimental animal model to mimic the presentation of TOF with its inherent physiologic variability in pressure- and volume-overload sequences, has not yet been elaborated.

According to our previous work, volume overload by TAP reconstruction of the RVOT immediately affected the systolic RV function, with impaired myocardial 
TABLE 2. Basic echocardiographic data

\begin{tabular}{|c|c|c|c|c|c|}
\hline Variable & $\mathbf{R V H}+\mathbf{P I}$ & RVH & PI & Sham & $P$ value \\
\hline \multicolumn{6}{|l|}{ TV E-wave $(\mathrm{m} / \mathrm{s})$} \\
\hline Acute & $0.67 \pm 0.27$ & $0.80 \pm 0.12$ & $0.92 \pm 0.16$ & $0.83 \pm 0.16$ & .40 \\
\hline Chronic & $0.73 \pm 0.15$ & $0.67 \pm 0.10$ & $0.81 \pm 0.06$ & $0.89 \pm 0.15$ & .10 \\
\hline \multicolumn{6}{|l|}{ TV A-wave $(\mathrm{m} / \mathrm{s})$} \\
\hline Acute & $0.63 \pm 0.22$ & $0.53 \pm 0.16$ & $0.64 \pm 0.08$ & $0.43 \pm 0.07$ & .30 \\
\hline Chronic & $0.56 \pm 0.06$ & $0.55 \pm 0.15$ & $0.66 \pm 0.09$ & $0.56 \pm 0.05$ & .47 \\
\hline \multicolumn{6}{|l|}{ TV E/A ratio } \\
\hline Acute & $1.11 \pm 0.39$ & $1.66 \pm 0.64$ & $1.42 \pm 0.17$ & $1.95 \pm 0.23$ & .13 \\
\hline Chronic & $1.33 \pm 0.34$ & $1.27 \pm 0.32$ & $1.23 \pm 0.07$ & $1.60 \pm 0.26$ & .41 \\
\hline \multicolumn{6}{|c|}{ TV E-wave DCT (ms) } \\
\hline Acute & $257 \pm 50$ & $192 \pm 17$ & $178 \pm 45$ & $181 \pm 15$ & .05 \\
\hline Chronic & $254 \pm 47$ & $200 \pm 43$ & $202 \pm 64$ & $179 \pm 10$ & .11 \\
\hline \multicolumn{6}{|c|}{ TV E-wave DC slope } \\
\hline Acute & $2.7 \pm 1.3$ & $4.1 \pm 0.7$ & $5.5 \pm 2.3$ & $4.7 \pm 1.2$ & .12 \\
\hline Chronic & $2.9 \pm 0.7$ & $3.4 \pm 0.7$ & $4.2 \pm 1.1$ & $5.0 \pm 0.7$ & $.01 *$ \\
\hline \multicolumn{6}{|c|}{ Restrictive pattern (n) } \\
\hline Acute & $4 / 6$ & $1 / 6$ & 0 & 0 & .04 \\
\hline Chronic & $5 / 6$ & 0 & 0 & 0 & .001 \\
\hline \multicolumn{6}{|l|}{ TAPSE (mm) } \\
\hline Acute & $13.0 \pm 1.4$ & $13.0 \pm 3.7$ & $13.7 \pm 2.1$ & $15.4 \pm 2.5$ & .67 \\
\hline Chronic & $17.4 \pm 4.3$ & $18.0 \pm 4.5$ & $14.3 \pm 2.9$ & $18.0 \pm 1.7$ & .58 \\
\hline \multicolumn{6}{|l|}{ FS $(\%)$} \\
\hline Acute & $37.3 \pm 5.6$ & $38.8 \pm 5.7$ & $37.5 \pm 0.5$ & $33.1 \pm 6.6$ & .55 \\
\hline Chronic & $33.0 \pm 10.5$ & $37.8 \pm 6.1$ & $30.7 \pm 4.2$ & $38.9 \pm 7.2$ & .38 \\
\hline \multicolumn{6}{|l|}{ PI grade } \\
\hline Acute & $3.4 \pm 0.5$ & $0.8 \pm 0.8$ & $3.7 \pm 0.6$ & 0 & $<.0001$ \\
\hline Chronic & $3.5 \pm 0.8$ & $0.6 \pm 0.8$ & $3.7 \pm 0.6$ & 0 & $<.0001$ \\
\hline \multicolumn{6}{|l|}{ PI duration (ms) } \\
\hline Acute & $256 \pm 14$ & 0 & $291 \pm 10$ & 0 & .01 \\
\hline Chronic & $265 \pm 19$ & 0 & $293 \pm 9$ & 0 & .05 \\
\hline \multicolumn{6}{|l|}{$\mathrm{RV}$ size $\left(\mathrm{mm} / \mathrm{m}^{2}\right)$} \\
\hline \multicolumn{6}{|l|}{ Basal level } \\
\hline Acute & $43.6 \pm 9.1$ & $41.3 \pm 4.7$ & $46.1 \pm 3.5$ & $34.7 \pm 2.6$ & .14 \\
\hline Chronic & $31.0 \pm 3.8$ & $29.3 \pm 4.4$ & $34.6 \pm 5.4 \ddagger$ & $24.0 \pm 3.8$ & .04 \\
\hline \multicolumn{6}{|c|}{ Midventricular level } \\
\hline Acute & $35.4 \pm 7.8$ & $32.0 \pm 2.9$ & $39.8 \pm 3.5$ & $32.9 \pm 0.6$ & .19 \\
\hline Chronic & $26.2 \pm 4.6$ & $24.6 \pm 2.8$ & $33.2 \pm 7.1$ & $23.4 \pm 5.3$ & .06 \\
\hline \multicolumn{6}{|l|}{ RVOT level } \\
\hline Acute & $28.9 \pm 5.8$ & $26.7 \pm 6.7$ & $29.9 \pm 5.0$ & $27.5 \pm 5.4$ & .88 \\
\hline Chronic & $21.3 \pm 3.4$ & $18.1 \pm 2.9$ & $26.2 \pm 3.6 \S$ & $19.8 \pm 4.5$ & .008 \\
\hline \multicolumn{6}{|l|}{ FWTI $\left(\mathrm{mm} / \mathrm{m}^{2}\right)$} \\
\hline Acute & $13.2 \pm 1.9$ & $12.5 \pm 2.8$ & $9.1 \pm 0.2$ & $8.9 \pm 1.4$ & $.02 \|$ \\
\hline Chronic & $9.5 \pm 1.0$ & $8.0 \pm 0.6$ & $7.0 \pm 0.6$ & $5.6 \pm 0.6$ & $<.0001$ \\
\hline \multicolumn{6}{|l|}{ IVST $\left(\mathrm{mm} / \mathrm{m}^{2}\right)$} \\
\hline Acute & $16.1 \pm 5.5$ & $13.6 \pm 3.4$ & $11.2 \pm 0.6$ & $8.2 \pm 0.8$ & .07 \\
\hline Chronic & $9.8 \pm 1.2$ & $8.0 \pm 1.3$ & $6.3 \pm 0.7$ & $7.0 \pm 0.9$ & $.003 \|$ \\
\hline
\end{tabular}

Analysis of variance used for all $P$ values; post hoc Tukey correction for multiple comparisons. $R V H$, Right ventricular hypertrophy; $P I$, pulmonary insufficiency; $T V$, tricuspid valve; $D C T$, deceleration time; $D C$, deceleration; TAPSE, tricuspid annular plane systolic excursion; $F S$, fractional shortening; $R V$, right ventricular; $R V O T$, RV outflow tract; FWTI, indexed free wall thickness; IVSTI, indexed interventricular septum thickness. $* P<.05$ between the sham and RVH+PI groups. $\dagger P<.05$ for all comparisons except between the sham and RVH groups and PI and RVH+PI groups. $\ddagger P<.05$ between the sham and PI groups. $\S P<.05$ between the PI group and the sham and RVH groups. $\| P<.05$ for all comparisons except between the RVH and RVH+PI groups and the PI and sham groups.

contractility through surgical damage of the infundibulum. ${ }^{6}$ Progression of PI involved significant RV dilation, with further deterioration of systolic function.

Pre-existing RVH appeared to alter this process in several ways. Previous pressure overload is known to initiate improved contractile performance by increasing the myocardial cell mass, merely by cell hyperplasia, such as has been shown in younger animals. ${ }^{4,9}$ In the present study, RVH yielded a contractile RV function comparable to that of the control pigs, despite significant PI in the 
acute phase. However, loss of contractile function was observed in relation to chronic volume overload, owing to secondary RV dilatation. RVH appeared to attenuate this phenomenon only partially. In contrast, when the right ventricle with hypertrophy was not physiologically challenged after relief of the obstruction, contractility was maintained.

RVH also influenced the remodeling secondary to chronic volume overload by retarding RV dilatation. This might have been related to the lower amount and shorter duration of PI, although the surgically created TAP was similar. Hence, it seemed more valid that both latter features were rather a consequence of the altered mechanical RV properties by hypertrophy than the primary cause. Kuehne and colleagues ${ }^{10}$ also stipulated the beneficial effect of $\mathrm{RVH}$ on the degree of PI. They found, in analogy, less RV dilation and enhanced myocardial contractility in an animal study by combining a component of stenosis to PI. Although they attributed RVH to be responsible for this effect, their experimental design had also involved a reduction of the regurgitant pulmonary orifice. However, it underscores the advantage of leaving an acceptable residual obstruction during relief of the RVOT to prolong the beneficial contribution of RVH on RV performance. ${ }^{11}$

In contrast to the favorable effect on myocardial contractility, hypertrophy has commonly been associated with decreased diastolic function, by impaired early relaxation and passive compliance. ${ }^{12}$ In this model, the determinants of early relaxation such as the minimal pressure-vs-time decline $\left(\mathrm{dP}_{\mathrm{d}} \mathrm{dT}_{\min }\right)$ and time-constant of pressure decay $(\tau)$ were not included, because these are known to be load dependent and poorly reliable in the context of PI-related disturbances of isovolumetric relaxation. However, RV compliance was significantly worse in both groups with RVH at both the acute and the chronic stage and was simultaneously associated with increased end-diastolic RV pressure. A restrictive RV physiology was noted only in the group combining RVH and significant PI, already present in the acute setting and persisting chronically. This finding highlights the mechanism of primary restriction, advancing the role of hypertrophy as its main substrate, and subsequently furthered by the volume overload by decreasing the diastolic pressure gradient between the stiff right ventricle and the pulmonary artery in the presence of significant pulmonary regurgitation.

Considering the histologic changes after 3 months, including microscopically prominent myocyte hypertrophy and interstitial fibrosis and, macroscopically, an increased mass/volume ratio, the advantage of $\mathrm{RVH}$ on $\mathrm{RV}$ remodeling after chronic volume overload might eventually be the combined result of sustained systolic performance and impaired diastolic properties of the right ventricle. One can assume that a stiffer hypertrophic ventricle, but with preserved contractility, would accommodate better to the PI and consequently resist longer the dilation process. This issue evokes the ambivalent role of a primary restrictive RV physiology in the clinical setting of TOF repair, with its detrimental effect on the early postoperative course, but its potentially favorable influence on late RV dilation. ${ }^{13,14}$

\section{Clinical Implications}

The present study has demonstrated that RVH entails a benefit on RV remodeling by chronic PI-related volume overload by affecting both systolic and diastolic RV performance. In TOF, a direct relationship exists between the extent of RVH and the duration of the pressure overload and, thus, the age of the patient. During the past decade, the age of TOF repair has been decreasing to even the neonatal age. Hence, this early repair policy has been accompanied by a frequent use of TAP, subjecting a right ventricle with less hypertrophy to chronic volume overload., ${ }^{2,3}$ This clinical situation corresponds to that of the PI group in our experiment, with the worst outcomes in terms of systolic function and RV dilatation. Munkhammar and colleagues ${ }^{15}$ revealed an inverse relationship between the presence of restrictive RV physiology and patient age at repair, irrespective of the type of RVOT reconstruction. A restrictive physiology was observed in only $10 \%$ of the patients $<6$ months old. ${ }^{15}$ In agreement with their doubts on the late effects of early repair, our study has supported the execution of primary repair after the neonatal age to take advantage of some degree of RVH, particularly in the patient whose RVOT morphology will require TAP repair for effective RVOT relief. Considering the risk of increased perioperative morbidity after repair in infants $<3$ months old, ${ }^{16}$ judiciously choosing a palliative shunt to postpone complete correction for a few months might be justified. However, the design of our experiment could not allow more precise advice on the optimal age for repair, because our study lacked variability in the quantification of RVH.

Otherwise, if RVOT morphology permits salvage of its functional integrity, early correction might be pursued, such as was shown in the RVH group, to overcome the incomplete recovery of diastolic compliance. Therefore, the ultimate answer to this conflicting problem concerning the timing and strategy of TOF repair requires an individualized approach to each patient, taking into account the specific RV-dependent characteristics, such as RVOT morphology and RVH.

\section{Study Limitations}

Although the present experimental model was relevant for its hemodynamic effects, extrapolation of these data to the clinical setting requires careful consideration because of the lack of confounding interaction with, specifically, 
cyanosis. This factor is often advanced by the duration and severity of RV pressure overload and, thus, the age of the TOF patient, and might additionally affect RV performance by enhancing some histologic alterations. Second, RVH was induced as a fixed component and precluded investigation of varying degrees of $\mathrm{RVH}$ on $\mathrm{RV}$ function. Additional study on this issue should include a wider period of previous pressure-overload exposure; however, this would be at the cost of a larger sample size of animals. Echocardiography was primarily used to validate the surgical result and included mainly basic measurements. The addition of tissue Doppler data might have allowed more subtle examination of the systolic and diastolic RV function, albeit its exact contribution remains vague in the setting of TOF.

\section{CONCLUSIONS}

In a model of juvenile growing swine, the role of RVH was studied for its effect on RV function and remodeling after acute and chronic PI-related volume overload. RVH initiated improved contractile function, which preserved global RV function longer in relation to chronic PI. However, it entailed decreased diastolic compliance, responsible for a restrictive RV physiology when simultaneously challenged by significant PI. Both alterations in $\mathrm{RV}$ performance affected secondary $\mathrm{RV}$ remodeling by retarding the dilation process and restricting the amount and duration of PI. From these findings, primary repair of TOF during the early postnatal period should not be favored if RVOT reconstruction has been definitely deemed to result in significant PI, prematurely loading a less hypertrophied right ventricle. These insights on the influence of RVH on chronic PI might help in the decision-making process regarding the timing and surgical strategy as an individualized process for each patient with TOF.

We thank Dr Ingrid Van Overbeke and Deborah Croes for the dedicated care of the animals during this particularly demanding experiment. We also thank Maria Olieslagers for her help in the organization of our study and for the technical assistance during each procedure.

\section{References}

1. Reddy VM, Liddicoat JR, McElhinney DB, Brook MM, Stanger P, Hanley FL. Routine primary repair of tetralogy of Fallot in neonates and infants less than three months of age. Ann Thorac Surg. 1995;60:S592-6.

2. Tamesberger MI, Lechner E, Mair R, Hofer A, Sames-Dolzer E, Tulzer G. Early primary repair of tetralogy of Fallot in neonates and infants less than four months of age. Ann Thorac Surg. 2008;86:1928-35.

3. Hirsch JC, Mosca RS, Bove EL. Complete repair of tetralogy of Fallot in the neonate: results in the modern era. Ann Surg. 2000;232:508-14.

4. Leeuwenburgh BP, Helbing WA, Steendijk P, Schoof PH, Baan J. Biventricular systolic function in young lambs subject to chronic systemic right ventricular pressure overload. Am J Physiol Heart Circ Physiol. 2001;281: H2697-704.

5. Gaynor SL, Maniar HS, Bloch JB, Steendijk P, Moon MR. Right atrial and ventricular adaptation to chronic right ventricular pressure overload. Circulation. 2005;112:I212-8.

6. Bove T, Bouchez S, De Hert S, Wouters P, De Somer F, Devos D, et al. Acute and chronic effects of dysfunction of right ventricular outflow tract components on right ventricular performance in a porcine model: implications for primary repair of tetralogy of Fallot. J Am Coll Cardiol. 2012;60:64-71.

7. Kelley KW, Curtis SE, Marzan GT, Karara HM, Anderson CR. Body surface area of female swine. J Animal Sci. 1973;36:927-30.

8. Redington AN, Oldershaw PJ, Shinebourne EA, Rigby ML. A new technique for the assessment of pulmonary regurgitation and its application to the assessment of right ventricular function before and after repair of tetralogy of Fallot. Br Heart J. 1988;60:57-65.

9. Leeuwenburgh BP, Helbing WA, Wenink AC, Steendijk P, de Jong R, Dreef EJ, et al. Chronic right ventricular pressure overload results in a hyperplastic rather than a hypertrophic myocardial response. J Anat. 2008;212:286-94.

10. Kuehne T, Gleason BK, Saeed M, Turner D, Weil J, Teitel DF, et al. Combined pulmonary stenosis and insufficiency preserves myocardial contractility in the developing heart of growing swine at midterm follow-up. J Appl Physiol. 2005;99:1422-7.

11. Yoo BW, Kim JO, Kim YJ, Choi JY, Park HK, Park YH, et al. Impact of pressure load caused by right ventricular outflow tract obstruction on right ventricular -volume overload in patients with repaired tetralogy of Fallot. J Thorac Cardiovasc Surg. 2012;143:1299-304.

12. Leeuwenburgh BP, Steendijk P, Helbing WA, Baan J. Indexes of diastolic RV function: load dependence and changes after chronic RV pressure overload in lambs. Am J Physiol Heart Circ Physiol. 2002;282:H1350-8.

13. Cullen S, Shore D, Redington A. Characterization of right ventricular diastolic performance after complete repair of tetralogy of Fallot: restrictive physiology predicts slow postoperative recovery. Circulation. 1995;91:1782-9.

14. Norgard G, Gatzoulis MA, Moraes F, Lincoln C, Shore DF, Shinebourne EA, et al. Relationship between type of outflow tract repair and postoperative right ventricular diastolic physiology in tetralogy of Fallot: implications for long-term outcome. Circulation. 1996;94:3276-80.

15. Munkhammar P, Cullen S, Jogi P, de Leval M, Elliott M, Norgard G. Early age at repair prevents restrictive right ventricular (RV) physiology after surgery for tetralogy of Fallot (TOF): diastolic RV function after TOF repair in infancy. J Am Coll Cardiol. 1998;32:1083-7.

16. van Dongen EI, Glansdorp AG, Mildner RJ, McCrindle BW, Sakopoulos AG VanArsdell G, et al. The influence of perioperative factors on outcomes in children aged less than 18 months after repair of tetralogy of Fallot. $J$ Thorac Cardiovasc Surg. 2003;126:703-10. 